\section{ESTUDO SOROLÓGICO DA TOXOPLAS. MOSE CANINA, PELA PROVA DE IMUNOFLUORESCENCIA INDIRETA, NA CIDADE DE CAMPINAS, 1981}

\author{
PEDRO MANOEL LEAL GERMANO \\ Professor Assistente Doutor
}

Faculdade de Medicina Veterinária e

Zootecnia da USP

\section{ELCIO BENEDITO ERBOLATO}

Auxiliar de Ensino

Faculdade de Medicina Veterinária e

Zootecnia da USP

MASAIO MLZUNO ISHLZUKA

Professora Adjunta

Faculdade de Medicina Veterinária e

Zootecnia da USP

GERMANO, P.M.L.; ERBOLATO, E.B.; ISHLZUKA, M.M. Estudo sorológico da toxoplasmose canina, pela prova de imunofluorescência indireta, na cidade de Campinas, 1981. Rev Fac.Med. vet.Zootec.Univ.S.Paulo, 22(1): 53-58, 1985.

RESUMO: A prevaléncia de infecção toxoplásmica em cães da cidade de Campinas, avaliada pela prova de imunofluorescência direta, revelou ser igual a $91 \%(88,8 \longmapsto 93,2 \%)$. Não foram observadas diferenças estatisticamente significantes entre as proporçōes de reagentes, quando considerados os sexos. Os diferentes grupos etários mostraram-se igulamente infectados e o nivel de anticorpos anti-toxoplasma mais frequentemente observado foi igual a 1.000 .

UNITERMOS: Toxoplasmose $^{+}$; Toxoplasma gondii, prevalência $^{+}$; Imunofluorescência indireta

\section{INTRODUÇĀO}

A toxoplasmose é uma moléstia parasitária de caráter zoonótico, provocada pelo Toxoplasma gondii e que na espécie humana assume grande importância, dada a gravidade com a qual se manifesta na forma congênita de transmissão (FRENKEL ${ }^{2}, 1971$; SCOTT $\left.^{7}, 1978\right)$. No cão, a exemplo do que ocorre no homem, a infecção é de evolução crônica, assintomática na grande maioria dos casos, mas que quando diante de condiçoes imunossupressoras manifesta-se clinicamente como uma moléstia grave.

No passado acreditava-se que a toxoplasmose fosse uma doença naturalmente transmissivel entre as diferentes espécies de animais e entre estas e o homem. A partir dos estudos de FRENKEL ${ }^{2}$ (1971), fundamentados no conhecimento do ciclo biológico do T.gondii, a cadeia de transmissão da moléstia foi melhor esclarecida. Assim, o homem e todos aqueles animais nos quais o $\mathbf{T}$. gondii tem sido descrito, com exceção dos felinos, são considerados como hospedeiros acidentais e, em alguns casos, como terminais, isto é, quando não ocorre a transmissão congênita.

Os alimentos vegetais contaminados com oocistos e os de origem animal, principalmente produtos suinos com cistos, são os maiores responsáveis pela infecção humana. No cão, além destes alimentos, estão envolvidos, ainda, o solo contaminado e roedores infectados, ingeridos parcial ou totalmente, como consequência do hábito de carnivorismo exercido por estes animais.

Do ponto de vista de Saúde Pública, a infecçâo na população canina significa que a área envolvida representa um nicho ecológico para o parasita e, conseqüentemente, um risco para a população humana.

Levantamentos soroepidemiológicos, realizados em animais da espécie canina, evidenciam a elevada prevalência com que a infeç̧ão ocorre nesta população. TURNER ${ }^{8}$ (1976) afirma que os valores de prevalência da toxoplasmose canina variam de 20 a $80 \%$, confirmando assim, ser esta infecçð̃o bastante disseminada.

ISHIZUKA et alii $^{4}$ (1974) e ISHIZUKA \& YASUDA $^{5}$ (1981) em Sđ̃o Paulo, em levantamentos sorológicos efetuados na população canina e realizados em épocas distintas, encontraram, respectivamente, $72,0 \%$ e $63,8 \%$ de prevalência de anticorpos anti-Toxoplasma gondii, confirmando também, que em nosso meio a toxoplasmose canina está bastante difundida.

Em razao destes resultados e com a finalidade de determinar os niveis de ocorrência da infecçđo por $\mathbf{T}$. gondii em cães de outros centros urbanos, elegeu-se para a realização do presente trabalho, a cidade de Campinas, segunda metróple do estado de São Paulo, com 750.000 habitantes e uma população canina estimada em 75.250 animais.

Destarte, o estudo em apreço objetiva avaliar a frequência de ç̃es reagentes à prova de imunofluorescência indireta para toxoplasmose e, ainda, ao teste das seguintes hi- 
póteses de nulidade:

1) não existência de diferença estatisticamente significante na proporção de reagentes à prova de imunofluorescência indireta, segundo o sexo;

2) ño diferença estatisticamente significante na proporção de reagentes pelos diferentes grupos etários;

3) nao existência de diferença na proporção de ocorrência dos diferentes valores dos títulos de anticorpos anti-Toxoplasma gondii em soros de cães.

É propósito do trabalho rejeitar as hipóteses acima citadas ao nivel de rejeiçáo de $5 \%$.

\section{MATERIAL E MÉTODOS}

Animais

Durante a Campanha de Vacinação Anti-rábica realizada em Campinas, de 17 a 22 de agosto de 1981, foram selecionados ao acaso, 13 postos de vacinação, tomando-se as devidas precauçסes para que os mesmos se distanciassem equitativamente, abrangendo toda a cidade em estudo.

Deste modo, de cada posto coletou-se sangue de cada 10 animais apresentados para a vacinaçáo anti-rábica, independente de raça e porte e considerados como restrições sexo e idade aproximada.

Desta forma, coletaram-se 657 amostras de sangue de cáes que, após devidamente tratadas no laboratório, forneceram igual quantidade de amostras de soro.

\section{Prova sorológica}

Utilizou-se a prova de imunofluoresciència indireta segundo a técnica adaptada por ISHIZUKA et alii ${ }^{3}$ (1974).

\section{Método estatístico}

O procedimento estatístico baseou-se na estimativa por intervalo da verdadeira proporção de cđ̃es reagentes à toxoplasmose para um limite de confiança igual a $95,0 \%$ (LESER et aliii 6 , 1973) e no teste da diferença entre duas porporções com aproximação normal (BERQUÓ et alii ${ }^{1}$, 1981).

\section{RESULTADOS}

Preliminarmente, constatou-se que dos 657 soros examinados, 598 foram positivos a uma diluiçao de pelo menos 1: 16 à prova de imunofluorescência indireta, revelando ser de $91,0 \%$ a estimativa da prevalência da infecçăo toxoplásmica em caes da cidade de Campinas. Uma vez submetido este valor de prevaiencia à estimativa por intervalo para 95,0\% de Confiança de conter pelo menos um animal reagente, obteve-se um intervalo igual a:
Limite de Confiança $(88,8 \% \longmapsto 92,3 \%) 95,0 \%$

A seguir, construiu-se a Tab. 1, onde se apresenta a frequência de animais reagentes e não reagentes à prova de imunofluorescência indireta segundo o sexo, na qual se pode observar que $93,0 \%$ dos reagentes pertencem ao sexo masculino e $86,7 \%$ ao sexo feminino. Os dados desta Tabela foram tratados estatisticamente, através da aplicação do teste de duas proporçōes com aproximação normal, que forneceu para a estatística $Z$ um valor igual a 2,60 , o qual se revelou significante para o nível de rejeição adotado.

$\mathrm{Na}$ Tab. 2 encontram-se os valores pertinentes aos resultados da prova de imunofluorescência indireta segundo a condição de reagentes ou não reagentes, classificados quanto à idade aproximada. Com base nos dados obtidos para os animais reagentes e através da utilização do teste de duas proporçoes, calculado para todas as faixas etárias, não houve diferença estatisticamente significante entre as proporçбes dos diversos grupos etários ao nivel de rejeição adotado.

A Tab. 3 apresenta os resultados da prova de imunofluorescência indireta dos animais reagentes, levando-se em consideração os diferentes títulos de anticorpos, classifica. dos segundo o grupo etário. Para a verificação do título de anticorpo anti-Toxoplasma mais frequente, constituiu-se a Tab. 4 que revelou ser a mediana igual a 1000 .

\section{DISCUSSĀO}

Avaliação quantitativa de ocorrência de infeç̧ăo toxoplásmica no homem e diferentes espécies animais tem despertado o interesse dos epidemiologistas e, neste contexto, o cão, animal de estimação preferencial do homem, representa objeto de indagação epidemiológica. Isto se deve ao fato de tanto o homem quanto os cães estarem, em muitas oportunidades, expostos ao mesmo risco de infecção representado pelo "habitat" e hábitos alimentares de camivorismo.

Assim, a prevalência da infecção que é a expressão da magnitude de ocorrência da mesma, no Município de Campinas, em agosto de 1981, foi igual a $91.0 \%$ e que para $95,0 \%$ de confiança podemos dizer que se encontra entre os valores 88,8 e $93,2 \%$.

$O$ valor encontrado neste experimento é consideravelmente superior ao encontrado por ISHIZUKA \& YASUDA $^{5}$, em São Paulo, em 1981, que fora igual a $63,8 \%$. Este último trabalho revelou nao haver diferença significante na ocorrência da infecção quando considerados os animais agrupados segundo o sexo, o que foi também comprovado por nós. Este último aspecto é facilmente compreendido, visto estar a transmissão da toxoplasmose relacionada com a ingestão de alimentos contaminadcs com T. gondii. 
Quando considerados os grupos etários, observou-se que os animais, desde a mais tenra idade, já se mostravam reagentes à imunofluorescência indireta, não sendo possível estabelecer-se uma idade limiar a partir da qual a porcentagem de infectados seria significantemente maior.

Relativamente aos niveis de anticorpos, verificou-se pelo cálculo da mediana, que o valor do título de anticorpos mais frequente é igual a 1000 . Este valor, juntamente com o valor da prevalência observada $91,0 \%$, permitem concluir que, além da infecção toxoplásmica ocorrer com elevada frequência, também se manifesta com elevada intensidade.

Os dados encontrados neste experimento sugerem que a infecção deve ser estudada neste município, do ponto de vista epidemiológico mais abrangente, a frm de se elucidarem as razôes de tão elevada prevalência.

\section{CONCLUSOESS}

1) A prevaléncia de reagentes à toxoplasmose pela prova de imunofluorescência indireta, em cães da cidade de Campinas, foi igual a $91,0 \%$, e que para $95,0 \%$ de confiança, encontrava-se entre os valores de 88,8 e $93,2 \%$.

2) Não houve diferença estatisticamente significante na proporção de reagentes entre os sexos.

3) Os animais mostraram-se reagentes desde a mais tenra idade (menos de 1 ano).

GERMANO, P.M.L.; ERBOLATO, E.B.; ISHIZUKA, M.M. Serological study of canine toxoplasmosis through indirect immunofluorescent technique. Rev.Fac.Med.vet.Zootec.Univ. S.Paulo, 22(1):53-58, 1985 .

SUMMARY: The prevalence of canine toxoplasmosis in dogs of Campinas city, evaluated through the indirect immunofluorescent technique, was $91.0 \%(88,8 \longrightarrow 93,2 \%)$. Considering the sex of the animalsy statistical significant differences were not observed among the proportion of reagents; further animals of different ages revealed the same infection rate. The most frequent value of anti-toxoplasma antibody titer observed was 1.000 .

UNITERMES: Toxoplasmosis ${ }^{+}$; Toxoplasma gondii, prevalence ${ }^{+}$; Immunofluorescent techniques ${ }^{+}$

TABELA 1 - Animais da espécie canina, segundo os resultados da prova de Imunofluorescência indireta para avaliação de anticorpos anti-Toxoplasma gondii e sexo. Campinas, SP, 1981.

\begin{tabular}{l|c|c|c|c|c}
\hline \multirow{2}{*}{ Sexo } & \multicolumn{2}{|c}{} & \multicolumn{2}{c}{ Não reagentes } & \multirow{2}{*}{ Total } \\
\cline { 2 - 6 } & Reagentes & F & $\%$ & \\
\hline Masculino & 422 & 93,0 & 32 & 7,0 & 454 \\
\hline Feminino & 176 & 86,7 & 27 & 13,3 & 203 \\
\hline Total & 598 & 91,0 & 59 & 9,0 & 657 \\
\hline $\begin{array}{l}\text { F = Frequência } \\
\%=\text { Porcentagem }\end{array}$ & & & & \\
\end{tabular}


TABELA 2 - Animais da espécie canina, segundo os resultados da prova de Imunofluorescência indireta para avaliação de anticorpos anti-Toxoplasma gondii e idade. Campinas, SP, 1981.

\begin{tabular}{|c|c|c|c|c|c|}
\hline & \multicolumn{2}{|c|}{ Reagentes } & \multicolumn{2}{|c|}{ Não reagentes } & \multirow{2}{*}{ Total } \\
\hline & F & $\%$ & $\mathrm{~F}$ & $\%$ & \\
\hline$<1$ & 61 & 75,3 & 20 & 24,7 & 81 \\
\hline $1 \longrightarrow 2$ & 117 & 86,7 & 18 & 13,3 & 135 \\
\hline $2 \longrightarrow 3$ & 106 & 93,0 & 8 & 7,0 & 114 \\
\hline $3 \longrightarrow 4$ & 123 & 94,6 & 7 & 5,4 & 130 \\
\hline $4 \longrightarrow 5$ & 62 & 95,4 & 3 & 4,6 & 65 \\
\hline $5 \longrightarrow 6$ & 43 & 97,7 & 1 & 2,3 & 44 \\
\hline $6 \longrightarrow 7$ & 32 & 100,0 & - & - & 32 \\
\hline $7 \longrightarrow 8$ & 16 & 100,0 & - & - & 16 \\
\hline $8 \longrightarrow 9$ & 19 & 100,0 & - & - & 19 \\
\hline $9 \longrightarrow 10$ & 5 & 100,0 & - & - & 5 \\
\hline$>10$ & 14 & 87,5 & 2 & 12,5 & 16 \\
\hline Total & 598 & 91,0 & 59 & 9,0 & 657 \\
\hline
\end{tabular}

$\mathrm{F}=$ Frequência

$\%=$ Porcentagem 


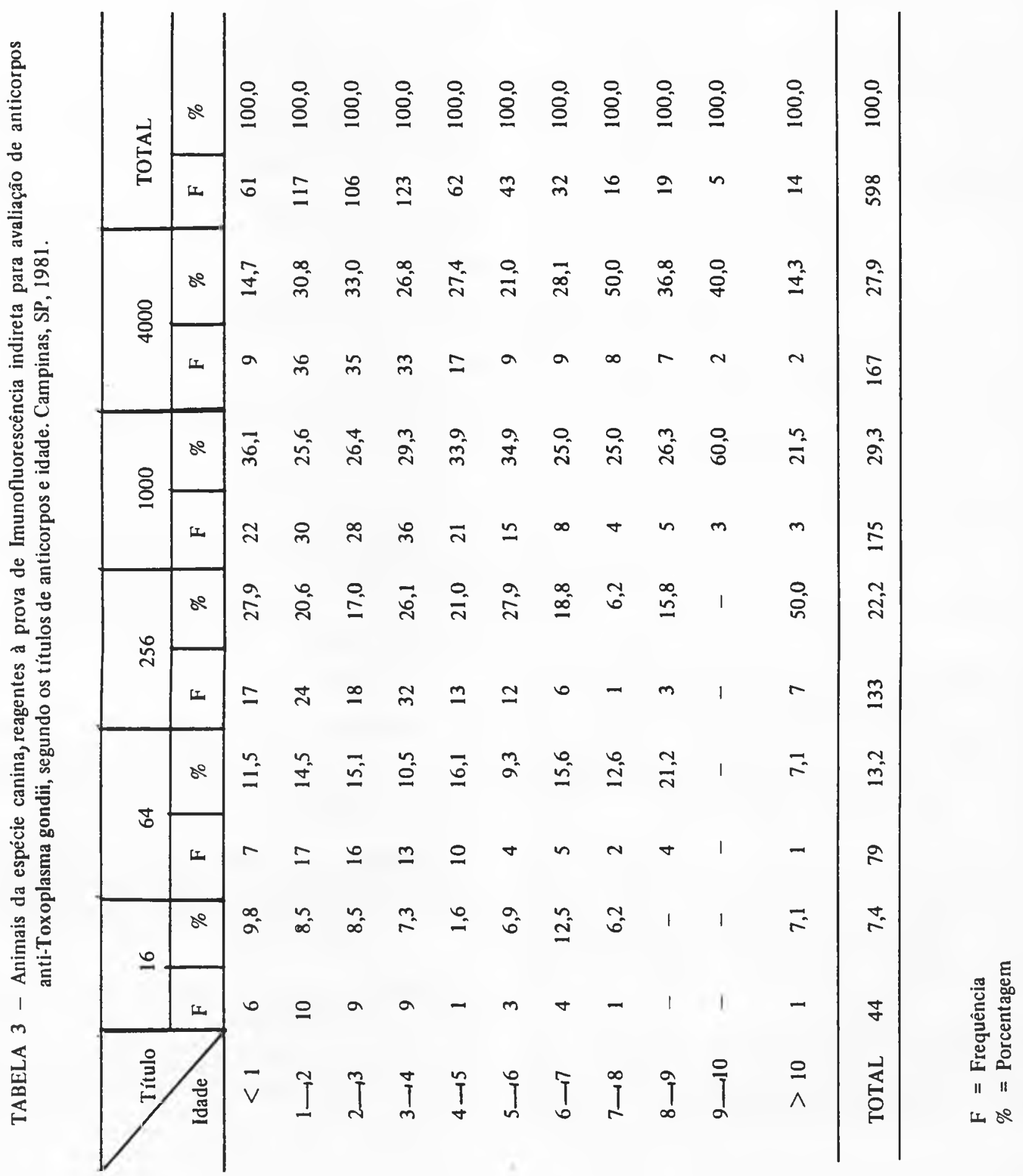


TABELA 4 - Animais da espécie canina, reagentes à prova de Imunofluorescência indireta para a a avaliação de anticorpos anti-Toxoplasma gondii, segundo a frequência e títulos de anticorpos. Campinas, SP, 1981.

\begin{tabular}{c|c|c|c}
\hline 16 & Absoluta & $\begin{array}{c}\text { Relativa } \\
(\%)\end{array}$ & Acumulada \\
\hline 64 & 44 & 7,4 & 44 \\
256 & 79 & 13,2 & 123 \\
1000 & 133 & 22,2 & 256 \\
4000 & 175 & 29,3 & 431 \\
Totalos & 598 & 27,9 & 598 \\
\hline F = Frequência & 100,0 & - \\
\hline
\end{tabular}

\section{REFERENCLIAS BIBLIOGRÄFICAS}

1 - BERQUÓ, E.S.; SOUZA, J.M.P.; GOTLIEB, S.L.D. Bioestatística. São Paulo, E.P.U., 1981.

2- FRENKEL, J.K. Toxoplasmosis: mechanisms of infection, laboratory diagnosis and management. Curr. Top. Path., 54:28-75, 1971.

3- ISHIZUKA, M.M.; MIGUEL, O.; BROGLIATO, D.F. Estudo comparativo entre as provas de SabinFeldman e imunofluorescência indireta para a avaliaçāo de anticorpos anti-toxoplasma em soros de çes. Rev.Fac.Med.vet.Zootec.Univ.S.Paulo, $11: 127-32,1974$.

4- ISHIZUKA, M.M.; MIGUEL, O.; BROGLIATO, D.F. Prevaléncia de anticorpos anti-toxoplasma em soros de çes do municipio de São Paulo. Rev. Fac.Med.vet.Zootec.Univ.S Paulo, 11:115-25,
1974.

5- ISHIZUKA, M.M. \& YASUDA, P.N. Incidència de infecção por Toxoplasma gondii em çes do município de São Paulo. Rev.Fac.Med.vet.Zootec. Univ.S.Paulo, 18:161-5, 1981.

6- LESER, W.; RIBEIRO NETTO, A.; GERMEK, O.A.; MARLET, J.M. Elementos de estatistica para a área de ciências da saúde. São Paulo, Escola Paulista de Medicina, 1973. (Apostila)

7- SCOTT, R.J. Toxoplasmosis. Bur. Hyg. and Trop. Dis., 75:809-27, 1978.

8- TURNER, G.V.S. Toxoplasmosis as a public health hazard. J. S. Afr. vet. Assoc., 47:227-31, 1976. 